2

\section{Virulence of beta-hemolytic streptococci in infective endocarditis}

2 Yvon RUCH ${ }^{1 \#}$, Yves HANSMANN ${ }^{1}$, Philippe RIEGEL ${ }^{2}$, Nicolas LEFEBVRE ${ }^{1}$, Jean-

3 Philippe MAZZUCOTELLI ${ }^{3}$, Nawal DOUIRI $^{1}$, Aurélie MARTIN $^{1}$, Xavier ARGEMI $^{1}$

$4{ }^{1}$ Department of Infectious Disease, Strasbourg University Hospital, Strasbourg, France

$5 \quad{ }^{2}$ Laboratory of Bacteriology, Strasbourg University Hospital, Strasbourg, France

$6{ }^{3}$ Department of Cardiovascular Surgery, Strasbourg University Hospital, Strasbourg, France

7

$8 \quad$ \#Corresponding author: yvon.ruch@gmail.com

9 Service de Maladies Infectieuses et Tropicales, Centre Hospitalo-Universitaire de Strasbourg

101 place de l'hôpital, BP 426, 67091 Strasbourg cedex, France

11 Phone: +33 3695512 19/Fax: +33 369551836

12 ORCID Id : 0000-0002-4713-9478

16 Running Title

17 Virulence of streptococci in endocarditis

18 Text word count: 2212

19 Abstract word count: 244 


\section{Abstract}

21 Background Streptococci involved in infective endocarditis (IE) primarily comprise alpha- or non-hemolytic streptococci (ANHS). Moreover, beta-hemolytic streptococci (BHS) can be involved, and guidelines recommend the addition of gentamicin for the first 2 weeks of treatment and the consideration of early surgery in such cases. This study compared the morbidity and mortality associated with IE depending on the microorganisms involved (BHS, ANHS, staphylococci and enterococci).

Methods We conducted a retrospective observational study between 2012 and 2017 in a single hospital in France. The endpoints were overall in-hospital mortality, 1-year mortality and the occurrence of complications.

Results We analyzed 316 episodes of definite IE including 150 (38\%), 96 (25\%), 46 (12\%) and 24 cases $(6 \%)$ of staphylococcal, ANHS, enterococcal and BHS IE, respectively. Inhospital mortality was significantly higher in the staphylococcal $(n=40 ; 26.7 \%)$ and BHS groups $(\mathrm{n}=6 ; 25.0 \%)$ than in the ANHS $(\mathrm{n}=9 ; 9.4 \%)$ and enterococcal groups $(\mathrm{n}=5$; $10.9 \%)($ all $p<0.01)$. The rates of septic shock and cerebral emboli were also higher in the BHS group than in the ANHS group [n $=7(29.2 \%)$ vs. $n=3(3.1 \%), p<0.001 ; n=7$ $(29.2 \%)$ vs. $\mathrm{n}=12(12.5 \%) ; \mathrm{p}=0.05$, respectively].

Conclusion This study confirmed that BHS IE has a more severe prognosis than ANHS IE. The virulence of BHS may be similar to that of staphylococci, justifying increased monitoring of these patients and more 'aggressive' treatments such as early surgery.

\section{Keywords}

Infective endocarditis; streptococci; beta-hemolytic; virulence; cardiac surgery 
 \\ Introduction}

43 Streptococci, which have long been implicated in most cases of infective endocarditis (IE),

$47[3]$

48 Alpha-hemolytic and non-hemolytic streptococci (ANHS) account for most of the streptococci involved in IE [4], whether their presumed origin is oral (mitis-oralis group) or and its management has not been clearly defined [5]. Considering the high virulence of group $\mathrm{B}, \mathrm{C}$ and $\mathrm{G}$ streptococci, recent guidelines recommend the addition of gentamicin for the first 2 weeks of treatment despite a low level of evidence $[5,6]$.

Thus, this study aimed to compare the characteristics of IE depending on the microorganisms involved, focusing on the mortality and morbidity associated with ANHS and BHS infections.

\section{Methods}

\section{Study design}

This retrospective study was conducted in the Strasbourg University Hospital, which is considered to be a reference center for the management of IE for a large region in France with approximately 70 cases per year. Data were extracted from a centralized database that stores medical information and therapeutic decisions of all patients with a final diagnosis of IE. Matrix-assisted laser desorption ionization-time of flight mass spectrometry was used determining the causative species. 
All patients diagnosed with definite IE according to modified Duke's criteria, in line with the

652015 European Society of Cardiology guidelines [5], involving staphylococci, streptococci or enterococci between January 2012 and December 2017, were included. Patients with pneumococcal IE and those with IE due to more than one microorganism were excluded.

Patients aged less than 18 years and those with ventricular assist devices were also excluded.

The primary endpoint of the study was overall in-hospital mortality rate. The secondary endpoints were the 1-year mortality rate occurrence of complications (septic shock, heart failure and stroke).

\section{Definitions}

The ANHS group included S. bovis complex, S. mitis complex, S. mutans complex, nutritionally variant streptococci (Abiotrophia and Granulicatella) and other non-classifiable non-BHS. The BHS group included $S$. agalactiae complex, $S$. dysgalactiae complex, $S$. pyogenes complex and $S$. anginosus complex, considering these latter as capable of betahemolysis and in line with the 2015 European Society of Cardiology guidelines [5]. The duration of antibiotic therapy was defined as the duration of appropriate treatment, in accordance with guidelines [5]. Septic shock was defined as a serum lactate level $>2 \mathrm{mmol} / \mathrm{L}$ and the requirement for a vasopressor to maintain a mean arterial pressure $\geq 65 \mathrm{mmHg}$, in accordance with the Sepsis-3 definition [7]. Time to surgery was defined as the time from the day of definite diagnosis of IE (first day when a 'definite diagnosis' according to modified Duke's criteria was met) to the day of the surgical procedure. Late surgery (surgery after the end of antibiotic therapy) was excluded from this definition. Time to diagnosis was defined as the time from the day (actual or presumed) from symptom onset to the day of definite diagnosis. Previous heart disease was defined as any type of heart disease, including valvular heart disease. 
90

\section{Statistical methods}

89 The descriptive analysis of continuous data was performed by obtaining the location parameters (i.e., mean, median, minimum, maximum, first and third quartiles) and the dispersion parameters (i.e., variance, standard deviation, range, interquartile range) for each variable. The Gaussian distribution of the data was tested using the Shapiro-Wilk test and quantile-quantile plots. The categorical data were described by obtaining the numbers and proportions for each category in the sample. Whenever useful, cross-tabulations were produced for each box of the table, which included numbers and proportions by row and column, as well as relative to the total. The inferential analysis for the categorical data was performed using the $\chi^{2}$ test or Fisher's exact test, depending on the theoretical size of the samples. Continuous data were compared using Student's $t$-test (Gaussian distribution of the variable) or a non-parametric test in the opposite case (Mann-Whitney $U$ test). Analysis of variance was used for continuous variables. The analyses were performed using R software

version 3.4.3.

\section{Ethical statements}

This study was approved by the Ethics Committee of Strasbourg University Hospital (registration number: FC/dossier 2017-36). According to French legislation (Jardé law, $\left.\mathrm{N}^{\circ} 2016-800\right)$, the non-opposition of all patients was sought.

\section{Results}

\section{Study population}

During the study period, 316 of 391 IE cases $(81 \%)$ in the database fulfilled the inclusion criteria, including 150 (38.4\%), 120 (30.7\%) and 46 cases (11.8\%) due to staphylococci, streptococci and enterococci, respectively (Fig. 1). Regarding streptococcal IE, 96 cases 
111 (80\%) involved ANHS, and 24 cases (20\%) involved BHS. Most ANHS IE involved

streptococci of the $S$. bovis (42 cases) or S. mitis group (41 cases). Over half of the BHS IE cases involved the S. agalactiae group (13 cases). More details on streptococcal species are available in the supplementary material (Supplementary Table S1).

115 The characteristics of patients are provided in Tables 1 and 2. The mean patient age was 65.8 \pm 15.5 years and 223 patients were male $(70.6 \%)$. Personal history and risk factors were comparable between the different microorganisms with the exception of intravenous drug users, who were overrepresented in the staphylococcal IE group.

Involvement of the aortic valve was most common (183 cases; $57.9 \%)$. This valve was affected more frequently in the ANHS and enterococci groups than in the BHS and staphylococci groups ( $\mathrm{p}<0.01)$. Overall, native valve IE was reported in 221 cases $(69.9 \%)$, followed by bioprosthetic valve IE (71 cases; $22.5 \%)$ and mechanical valve IE ( 24 cases; $7.6 \%)$

The number of positive blood culture bottles was significantly higher in the ANHS group than in the BHS group (3.0 vs. 1.9, $\mathrm{p}<0.0001)$. The mean serum C-reactive protein and median serum troponin levels were also significantly higher in the ANHS group than in the BHS group (208 mg/L vs. $110 \mathrm{mg} / \mathrm{L}, \mathrm{p}<0.0001 ; 0.44 \mu \mathrm{g} / \mathrm{L}$ vs. $0.07 \mu \mathrm{g} / \mathrm{L}, \mathrm{p}=0.01)$.

In comparison with the BHS group data, echocardiographic findings revealed significantly 129 more valvular perforations in the ANHS group (42 cases [43.8\%] vs. 4 cases [16.7\%], p < 130 0.01) and more cases of severe or important valve insufficiencies (54 cases [56.3\%] vs. 7 131 cases [29.2\%], $\mathrm{p}<0.001)$. The frequency of cerebral embolism did not differ significantly 132 between the microorganisms, although cerebral ischemia was numerically more frequent in 133 the BHS groups than in the ANHS groups (11 cases [61.1\%] vs. 26 cases [37.7\%], $p=0.08$ ). 
134 Surgery rates were significantly different among the four groups, with lower rates in the

staphylococci and BHS groups, but the difference was not statistically significant between the BHS and ANHS groups (11 cases [45.8\%] vs. 61 cases [63.5\%], p=0.11). Similar results were found regarding the duration of antibiotic therapy. Time to surgery and the length of inhospital stay did not differ significantly between the two groups. Time to diagnosis was shorter in the BHS group than in the ANHS group (17.7 days vs. 36.5 days, $\mathrm{p}<0.01)$. There was no difference regarding the use of aminoglycosides.

\section{Outcomes}

The in-hospital mortality rates were significantly higher in the staphylococci and BHS groups than in the ANHS and enterococci groups (26.7 and 25.0\%, respectively, vs. 9.4 and $10.9 \%$, respectively; all $\mathrm{p}<0.01$ ), although the difference did not reach significance between the BHS and ANHS groups (odds ratio $[\mathrm{OR}]=3.18,95 \%$ confidence interval $[\mathrm{CI}]=0.82-11.56$, $\mathrm{p}=0.08$ ) (Table 3). This difference persisted after 1 year of follow-up. Among the six deaths in the BHS group, five occurred in patients with $S$. agalactiae IE (mortality rate $=38.5 \%$ in this subgroup).

149 In comparison with the findings for the ANHS group, IE involving BHS was complicated 150 significantly more often with septic shock ( 7 cases [29.2\%] vs. 3 cases [3.1\%], OR $=12.35$, $15195 \% \mathrm{CI}=2.52-81.33, \mathrm{p}<0.001)$. Heart failure and stroke rates were also higher in the BHS 152 group, but these differences did not reach statistically significance.

\section{Discussion}

154 Our study revealed major differences between streptococci IE according to whether they are 155 beta-hemolytic. The in-hospital mortality rate was higher in the BHS IE group than in the 156 ANHS IE group, albeit without significance, whereas mortality rates were similar between the 157 BHS and staphylococci groups. S. agalactiae group (former known as Group B streptococci) 
was the predominant microorganism among BHS, as previously described $[8,9]$. This species was involved in five of six deaths in this group. Previous data found that the mortality rates in invasive BHS infections ranged from 11 to $21 \%$ [10-12] and from 25 to $27 \%$ in the largest BHS endocarditis series [9,13]. S. agalactiae IE appears to have the worse prognosis, with mortality rates of approximately 50\% [14,15]. Ivanova-Georgieva et al. recently compared $S$. aureus and $S$. agalactiae left-sided endocarditis and recorded similarly high mortality rates [16]

165 More complications occurred in the BHS group than in the ANHS group, and septic shock 166 rates (29\%) were similar to those reported for staphylococcal IE and higher than previously 167 reported findings $[13,16]$. Heart failure and stroke rates did not differ significantly between 168 the groups, but these conditions were more frequent in the BHS IE group. Cerebral ischemia 169 was observed in $61 \%$ of patients with BHS IE, which can be linked to the higher clinically 170 relevant stroke rate in this group. Nevertheless, the rate of heart failure in the BHS IE group 171 was surprising given the echocardiographic findings. Indeed, important or severe valve 172 insufficiency and valvular perforation were significantly less frequent in the BHS group than 173 in the other groups. Olmos et al. reported in 2015 high rates of valve insufficiency in 181 174 cases of S. bovis and viridans group streptococci IE [17]. We hypothesize that the longer time 175 to diagnosis after the onset of symptoms in the ANHS IE group than in the BHS or 176 staphylococcal IE group, as recently described [18], could have resulted in more valve 177 damage at the time of diagnosis. The increased frequency of damage in the ANHS IE group 178 could also be one explanation for the higher rate of surgery in this group, whereas the high 179 rate of complications in the BHS IE group might have delayed surgery because of the elevated 180 risk of perioperative death.

181 Most cases of BHS IE occurred in patients with apparently normal native valves, as only $33 \%$ 182 of patients had known heart disease and few patients had prosthetic valves (16.7\%), although 
these differences were not significant. Conversely, it appeared that ANHS IE occurred

184 predominantly with a disposition to previous valvulopathy [17] relative to BHS IE [8]. This

185 observation highlights the higher virulence of BHS than of ANHS. The more frequent

186 involvement of the aortic valve in ANHS IE could be explained by the greater multivalvular 187 involvement in this group (13 cases vs. none in the BHS group) and higher frequency of 188 prosthetic valve IE (mitral prosthetic valve IE is less common than aortic valve IE [19,20]).

189 The average number of positive blood culture bottles differed according to the causative 190 microorganism. The higher number of positive bottles was expected for staphylococci 191 considering their virulence and the difficulty of sterilizing these samples. We believe that the 192 difference observed between BHS and ANHS is linked to the time to diagnosis. Indeed, the 193 diagnosis was made almost twice as early for BHS compared to ANHS, probably because of 194 their more severe initial presentation.

195 Our study revealed a high proportion of patients receiving a combination therapy including 196 aminoglycosides, especially in the streptococci groups. European and American guidelines for 197 IE have recommended the adjunction of gentamicin for two weeks in BHS IE [5,6]. Some 198 authors recently proposed to abandon this recommendation in native valve IE given the lack 199 of evidence [21].

200 The present study was characterized by several strengths. Few data are available regarding 201 BHS endocarditis, and this study analyzed a large cohort to assess the virulence of 202 streptococci whether they were beta-hemolytic. In addition, our selection criteria were strict203 we only included 'definite IE'-and patients characteristics were consistent with the generally 204 observed epidemiology of IE in large cohort studies [4,22].

205 This work also had limitations. The observational and retrospective design may be the source 206 of biases inherent to this type of study. It was a single-center study; as such, the results might 
reflect some local habits, and they must be generalized with caution. Finally, the data are

208 limited by the rarity of BHS IE. As a result, our study lacks statistical power to demonstrate 209 small differences, which may explain why the primary outcome did not reach statistical 210 significance. We did not observe any Streptococcus pyogenes IE in our BHS group. This 211 bacterium is rarely involved in IE and most reported cases are in children [23,24], but only 212 adults were included in our study. We did not collect time-to-positivity of blood cultures.

213 In conclusion, BHS IE presented mostly as acute infections involving native and normal 214 valves with more severe complications such as cerebral embolism or septic shock and high 215 mortality rates. It appears that the virulence of BHS IE was similar to that of staphylococci, as 216 recently reported [16]. Surgery has been correlated with improved survival [25]. The 217 guidelines established in 2015 by the European Society of Cardiology recommend early 218 surgery in patients with $S$. aureus prosthetic valve IE and when favorable early response to 219 antibiotics is not achieved in patients with $S$. aureus native valve IE [5]. Thus, as suggested in 220 the guidelines, this study provides additional arguments in favor of proactive management of 221 BHS endocarditis. 
223 Conflict of interests There are no reported conflicts of interest.

229 JPM collected the data. YR and XA analyzed the data. PR analyzed the microbiological data.

230 YR performed the statistical analysis. YR and XA wrote the manuscript. All authors

231 reviewed, revised and approved the final report. 


\section{Slipczuk L, Codolosa JN, Davila CD, Romero-Corral A, Yun J, Pressman GS, et al.} Infective Endocarditis Epidemiology Over Five Decades: A Systematic Review. PLoS One. 2013;8:e82665.

2. Duval X, Delahaye F, Alla F, Tattevin P, Obadia J-F, Le Moing V, et al. Temporal Trends in Infective Endocarditis in the Context of Prophylaxis Guideline Modifications: Three Successive Population-Based Surveys. J Am Coll Cardiol. 2012;59:1968-76.

3. Sy RW, Kritharides L. Health care exposure and age in infective endocarditis: results of a contemporary population-based profile of 1536 patients in Australia. Eur Heart J. 2010;31:1890-7.

4. Murdoch DR, Corey GR, Hoen B, Miró JM, Fowler VG, Bayer AS, et al. Clinical Presentation, Etiology and Outcome of Infective Endocarditis in the 21st Century: The International Collaboration on Endocarditis-Prospective Cohort Study. Arch Intern Med. 2009;169:463-73.

5. Habib G, Lancellotti P, Antunes MJ, Bongiorni MG, Casalta J-P, Del Zotti F, et al. 2015 ESC Guidelines for the management of infective endocarditisThe Task Force for the Management of Infective Endocarditis of the European Society of Cardiology (ESC) Endorsed by: European Association for Cardio-Thoracic Surgery (EACTS), the European Association of Nuclear Medicine (EANM). Eur Heart J. 2015;36:3075-128.

6. Baddour LM, Wilson WR, Bayer AS, Fowler VG, Tleyjeh IM, Rybak MJ, et al. Infective Endocarditis in Adults: Diagnosis, Antimicrobial Therapy, and Management of Complications: A Scientific Statement for Healthcare Professionals From the American Heart Association. Circulation. 2015;132:1435-86. 
255 7. Singer M, Deutschman CS, Seymour CW, Shankar-Hari M, Annane D, Bauer M, et al. The

256 Third International Consensus Definitions for Sepsis and Septic Shock (Sepsis-3). JAMA. 257 2016;315:801-10.

258 8. Baddour LM. Infective endocarditis caused by beta-hemolytic streptococci. The Infectious 259 Diseases Society of America's Emerging Infections Network. Clin Infect Dis. 1998;26:66-71. 260 9. Lefort A, Lortholary O, Casassus P, Selton-Suty C, Guillevin L, Mainardi J-L, et al. 261 Comparison between adult endocarditis due to beta-hemolytic streptococci (serogroups A, B, 262 C, and G) and Streptococcus milleri: a multicenter study in France. Arch Intern Med. $2632002 ; 162: 2450-6$.

264 10. Ekelund K, Skinhøj P, Madsen J, Konradsen HB. Invasive group A, B, C and G 265 streptococcal infections in Denmark 1999-2002: epidemiological and clinical aspects. Clin 266 Microbiol Infect. 2005;11:569-76.

267 11. Rantala S, Vuopio-Varkila J, Vuento R, Huhtala H, Syrjänen J. Clinical presentations and 268 epidemiology of beta-haemolytic streptococcal bacteraemia: a population-based study. Clin 269 Microbiol Infect. 2009;15:286-8.

270 12. Oppegaard O, Mylvaganam H, Kittang BR. Beta-haemolytic group A, C and G 271 streptococcal infections in Western Norway: a 15-year retrospective survey. Clin Microbiol 272 Infect. 2015;21:171-8.

273 13. El Rafei A, DeSimone DC, DeSimone CV, Lahr BD, Steckelberg JM, Sohail MR, et al. 274 Beta-haemolytic streptococcal endocarditis: clinical presentation, management and outcomes. 275 Infect Dis. 2016;48:373-8. 
14. Sambola A, Miro JM, Tornos MP, Almirante B, Moreno-Torrico A, Gurgui M, et al.

277 Streptococcus agalactiae infective endocarditis: analysis of 30 cases and review of the 278 literature, 1962-1998. Clin Infect Dis. 2002;34:1576-84.

280 of Streptococcus agalactiae native valve endocarditis. Am Heart J. 2003;146:1095-8.

281 16. Ivanova-Georgieva R, Ruiz-Morales J, García-Cabrera E, García-López MV, Gálvez282 Acebal J, Plata-Ciezar A, et al. Left-sided infective endocarditis caused by Streptococcus 283 agalactiae: rare and serious. Eur J Clin Microbiol Infect Dis. 2019;38:265-75.

284 17. Olmos C, Vilacosta I, Sarriá C, López J, Ferrera C, Sáez C, et al. Streptococcus bovis 285 endocarditis: Update from a multicenter registry. Am Heart J. 2016;171:7-13.

286 18. Bläckberg A, Nilson B, Özenci V, Olaison L, Rasmussen M. Infective endocarditis due to 287 Streptococcus dysgalactiae: clinical presentation and microbiological features. Eur J Clin 288 Microbiol Infect Dis. 2018;37:2261-72.

289 19. Wang A, Athan E, Pappas PA, Fowler VG, Olaison L, Paré C, et al. Contemporary 290 Clinical Profile and Outcome of Prosthetic Valve Endocarditis. JAMA. 2007;297:1354-61. 291 20. Mahesh B, Angelini G, Caputo M, Jin XY, Bryan A. Prosthetic valve endocarditis. Ann 292 Thorac Surg. 2005;80:1151-8.

293 21. Sendi P. Infective endocarditis caused by Streptococcus agalactiae: time for beta294 hemolytic streptococci to follow treatment recommendations for S. aureus? Eur J Clin 295 Microbiol Infect Dis. 2019;38:419-22. 
22. Selton-Suty C, Célard M, Le Moing V, Doco-Lecompte T, Chirouze C, Iung B, et al.

1

2 3 4 5 6 7 9 10

297 Preeminence of Staphylococcus aureus in infective endocarditis: a 1-year population-based 298 survey. Clin Infect Dis. 2012;54:1230-9.

299 23. Day MD, Gauvreau K, Shulman S, Newburger JW. Characteristics of Children

300 Hospitalized With Infective Endocarditis. Circulation. 2009;119:865-70.

301 24. Weidman DR, Al-Hashami H, Morris SK. Two cases and a review of Streptococcus

302 pyogenes endocarditis in children. BMC Pediatr. 2014;14:227.

303 25. Chu VH, Park LP, Athan E, Delahaye F, Freiberger T, Lamas C, et al. Association

304 between surgical indications, operative risk, and clinical outcome in infective endocarditis: a 305 prospective study from the International Collaboration on Endocarditis. Circulation. $306 \quad 2015 ; 131: 131-40$. 


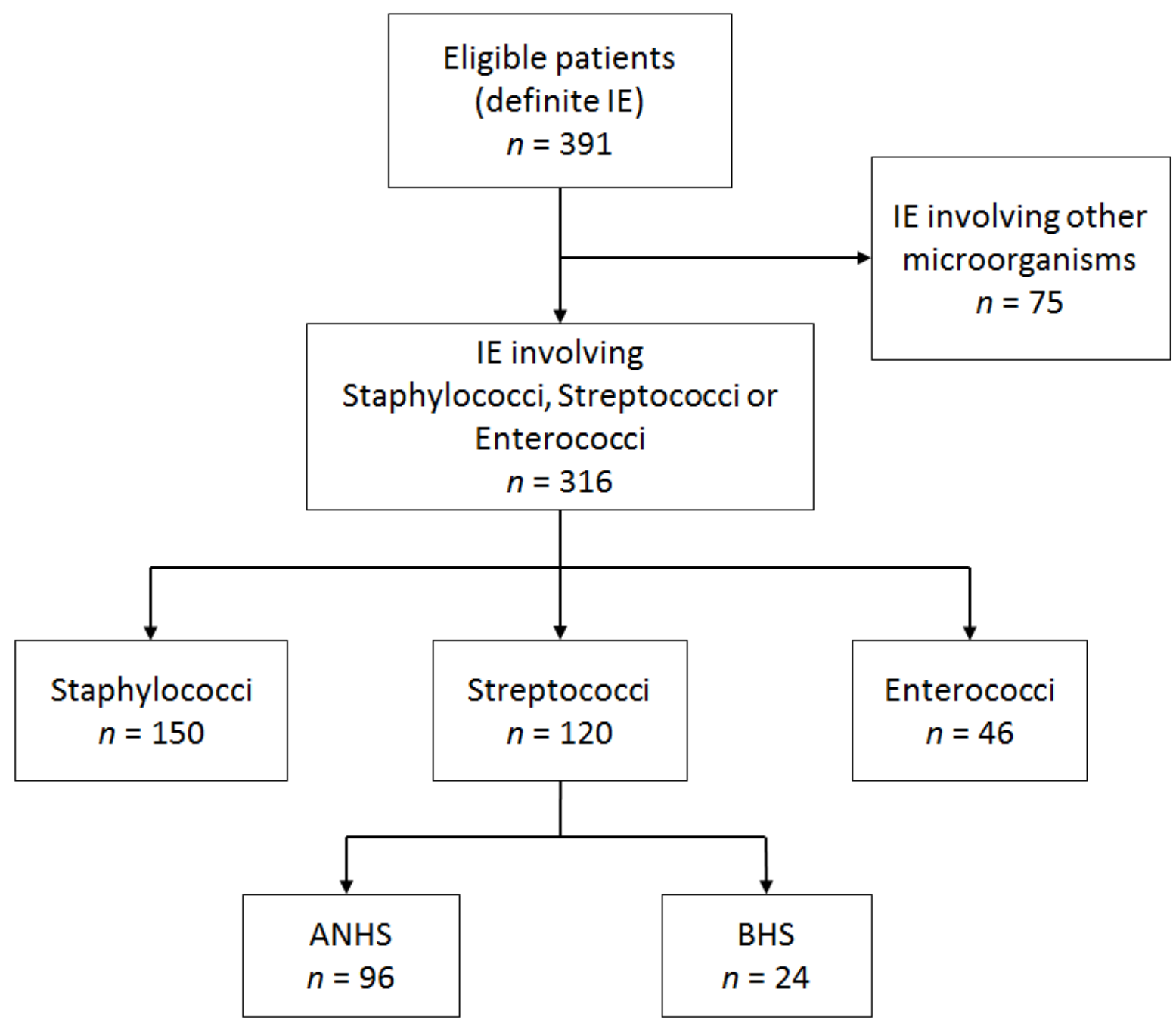

Fig. 1 Study flow chart

Abbreviations: IE, infective endocarditis; ANHS, alpha- or non-hemolytic streptococci; BHS, beta-hemolytic streptococci 
Table 1 Demographic characteristics and valve involvement among 316 patients with IE according to the causative microorganism

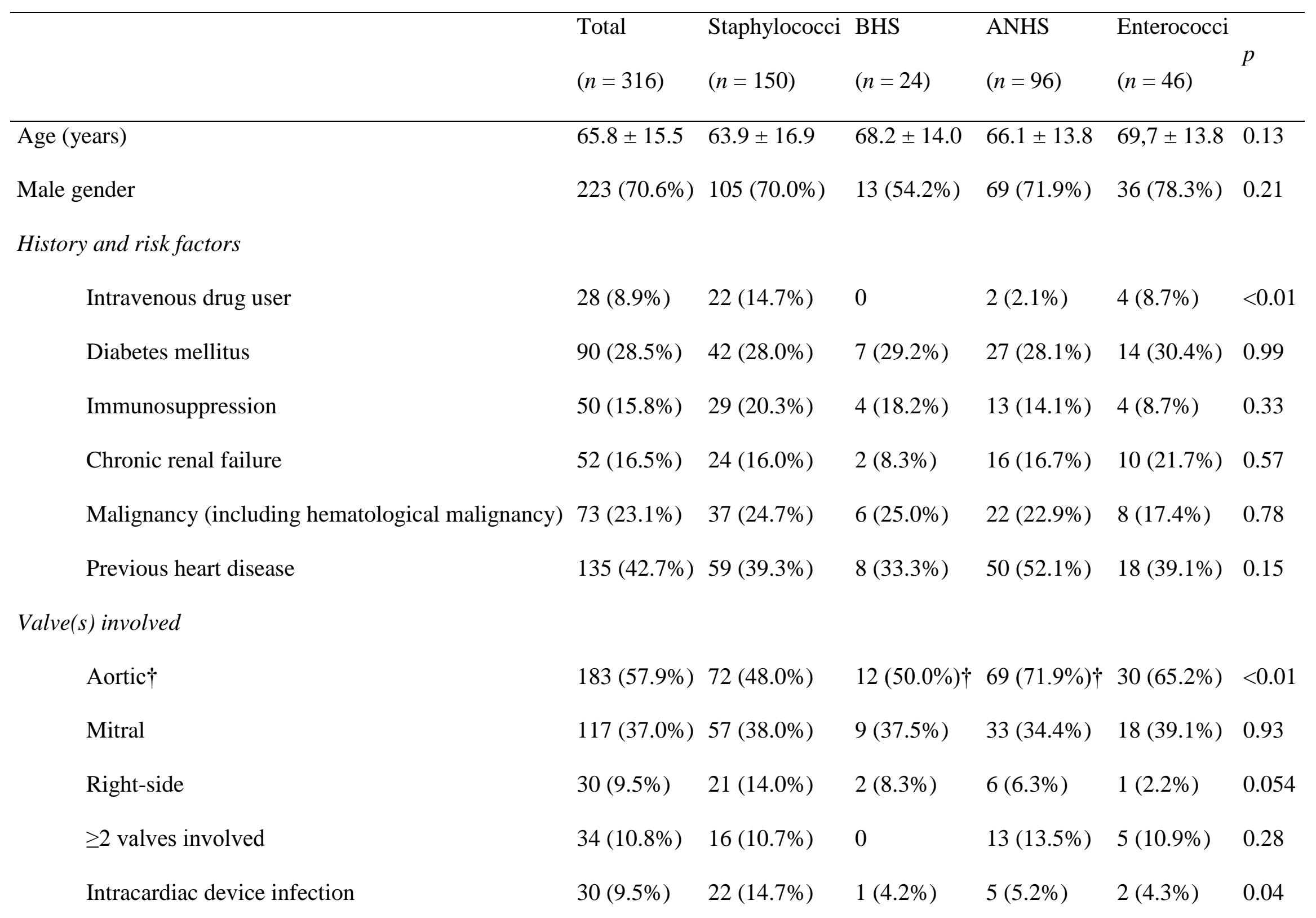


Native

Prosthetic

Bioprosthetic valve (including TAVR)

Mechanical valve
$221(69.9 \%) \quad 111(74.0 \%) \quad 20(83.3 \%) \quad 65(67.7 \%) \quad 25(54.3 \%)$

$95(30.1 \%) \quad 39(26.0 \%) \quad 4(16.7 \%) \quad 31(32.3 \%) \quad 21(45.7 \%)$

$71(22.5 \%) \quad 28(18.7 \%) \quad 3(12.5 \%) \quad 23(24.0 \%) \quad 17(37.0 \%)$

$24(7.6 \%) \quad 11(7.3 \%) \quad 1(4.2 \%) \quad 8(8.3 \%) \quad 4(8.7 \%)$

316

317 Values are presented as $\mathrm{n}(\%)$ or the mean \pm standard deviation, as appropriate.

$34318 \uparrow p<0.05$ for the comparison between BHS and ANHS

37319 Abbreviations: IE, infective endocarditis; BHS, beta-hemolytic streptococci; ANHS, alpha- or non-hemolytic streptococci; TAVR, transcatheter

39320 aortic valve replacement

40

41

42

43

45

46

47

48

49

50

51

52

53

54

55

56

57

58

59

60

61 
Table 2 Biological and echocardiographic findings, embolic events and management of 316 patients with IE according to the causative microorganism

\begin{tabular}{|c|c|c|c|c|c|c|}
\hline & $\begin{array}{l}\text { Total } \\
(n=316)\end{array}$ & $\begin{array}{l}\text { Staphylococci } \\
(n=150)\end{array}$ & $\begin{array}{l}\text { BHS } \\
(n=24)\end{array}$ & $\begin{array}{l}\text { ANHS } \\
(n=96)\end{array}$ & $\begin{array}{l}\text { Enterococci } \\
(n=46)\end{array}$ & $p$ \\
\hline \multicolumn{7}{|l|}{ Biological parameters } \\
\hline Number of positive blood culture bottles $\dagger$ & $3.3 \pm 2.1$ & $3.9 \pm 2.4$ & $1.8 \pm 1.6 \dagger$ & $3.0 \pm 1.6 \dagger$ & $2.8 \pm 1.6$ & $<0.0001$ \\
\hline $\begin{array}{l}\text { Number of positive blood culture bottles/number } \\
\text { of samples }\end{array}$ & $0.93 \pm 0.2$ & $0.96 \pm 0.1$ & $0.81 \pm 0.4$ & $0.9 \pm 0.2$ & $0.93 \pm 0.2$ & 0.02 \\
\hline Serum C-reactive protein level $(\mathrm{mg} / \mathrm{L}) \dagger$ & $174 \pm 102$ & $210 \pm 105$ & $208 \pm 130 \dagger$ & $110 \pm 51 \dagger$ & $127 \pm 59$ & $<0.0001$ \\
\hline Serum BNP level, median (ng/L) [IQR] & 511 [799] & $421[902]$ & $518[1128]$ & $447[661]$ & 799 [785] & 0.33 \\
\hline Serum troponin level, median $(\mu \mathrm{g} / \mathrm{L})[\mathrm{IQR}] \dagger$ & $0.18[0.9]$ & $0.22[1.1]$ & $0.44[8.3] \dagger$ & $0.07[0.24] \dagger$ & $0.17[0.9]$ & 0.01 \\
\hline \multicolumn{7}{|l|}{ Echocardiographic parameters } \\
\hline Maximal length of vegetation (mm) & $14.4 \pm 7.5$ & $15.4 \pm 8.4$ & $13.8 \pm 6.3$ & $13.6 \pm 6.5$ & $12.7 \pm 7.3$ & 0.23 \\
\hline Severe or important valve insufficiency $\dagger$ & $128(40.5 \%)$ & $47(31.3 \%)$ & $7(29.2 \%) \dagger$ & $54(56.3 \%) \dagger$ & $20(43.5 \%)$ & $<0.001$ \\
\hline Valvular perforation $\dagger$ & $95(30.0 \%)$ & $36(24.0 \%)$ & $4(16.7 \%) \dagger$ & $42(43.8 \%) \dagger$ & $13(28.3 \%)$ & $<0.01$ \\
\hline Periprosthetic regurgitation & $18(5.7 \%)$ & $6(15.4 \%)$ & $1(25.0 \%)$ & $5(16.1 \%)$ & $6(28.6 \%)$ & 0.53 \\
\hline Intracardiac abscess & $67(21.2 \%)$ & $32(21.3 \%)$ & $4(16.7 \%)$ & $15(15.6 \%)$ & $16(34.8 \%)$ & 0.07 \\
\hline \multicolumn{7}{|l|}{ Systemic embolism } \\
\hline Cerebral ischemia (on CT and/or MRI) $(\mathrm{n}=230)$ & $104(45.2 \%)$ & $51(45.9 \%)$ & $11(61.1 \%)$ & $26(37.7 \%)$ & $16(50.0 \%)$ & 0.33 \\
\hline
\end{tabular}




\section{Management}

\section{Time to diagnosis (days) $\dagger$}

Cardiac surgery

Time to surgical procedure (days)

Duration of antibiotic therapy (days)

Treatment including aminoglycosides

Duration of aminoglycoside therapy (days)

Length of in-hospital stay (days)

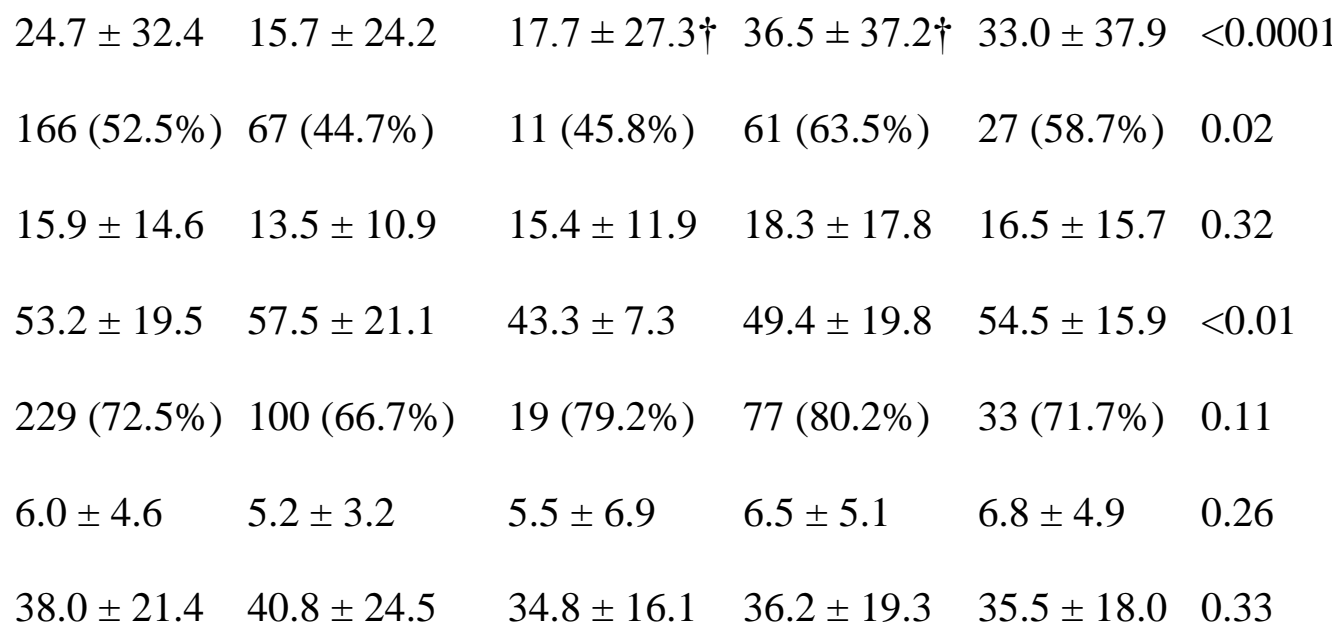

$\dagger p<0.05$ for the comparison between BHS and ANHS

Abbreviations: IE, infective endocarditis; BHS, beta-hemolytic streptococci; ANHS, alpha- or non-hemolytic streptococci; BNP, brain natriuretic

peptide; CT, computed tomography; MRI, magnetic resonance imaging; IQR, interquartile range 
Table 3 Complications and outcomes of 316 patients with IE according to the causative microorganism

\begin{tabular}{|c|c|c|c|c|c|c|c|c|c|}
\hline & $\begin{array}{l}\text { Total } \\
(n=316)\end{array}$ & $\begin{array}{l}\text { Staphylococci } \\
(n=150)\end{array}$ & $\begin{array}{l}\text { S. aureus } \\
(n=115)\end{array}$ & $\begin{array}{l}\text { BHS } \\
(n=24)\end{array}$ & $\begin{array}{l}\text { ANHS } \\
(n=96)\end{array}$ & $\begin{array}{l}\text { Enterococci } \\
(n=46)\end{array}$ & $P^{\#}$ & $p^{*}$ & $\begin{array}{l}\text { OR } \\
(95 \% \mathrm{CI})^{*}\end{array}$ \\
\hline $\begin{array}{l}\text { In-hospital } \\
\text { mortality }\end{array}$ & $60(19.0 \%)$ & $40(26.7 \%)$ & $34(29.6 \%)$ & $6(25.0 \%)$ & $9(9.4 \%)$ & $5(10.9 \%)$ & $<0.001$ & 0.08 & $\begin{array}{l}3.18 \\
(0.82-11.56)\end{array}$ \\
\hline $\begin{array}{l}\text { One-year } \\
\text { mortality }\end{array}$ & $67(21.2 \%)$ & $44(29.3 \%)$ & $37(32.2 \%)$ & $6(25.0 \%)$ & $10(10.4 \%)$ & $7(15.2 \%)$ & $<0.001$ & 0.09 & $\begin{array}{l}2.84 \\
(0.75-10.00)\end{array}$ \\
\hline Septic shock $\dagger$ & $57(18.0 \%)$ & $44(29.3 \%)$ & $41(35.7 \%)$ & $7(29.2 \%)$ & $3(3.1 \%)$ & $3(6.5 \%)$ & $<0.0001$ & $<0.001$ & $\begin{array}{l}12.35 \\
(2.52-81.33)\end{array}$ \\
\hline Heart failure & $169(53.5 \%)$ & $79(52.7 \%)$ & $63(54.8 \%)$ & $16(66.7 \%)$ & $51(53.1 \%)$ & $23(50.0 \%)$ & 0.60 & 0.23 & $\begin{array}{l}1.76 \\
(0.64-5.22)\end{array}$ \\
\hline $\begin{array}{l}\text { Clinically } \\
\text { significant stroke }\end{array}$ & $49(15.5 \%)$ & $27(18.0 \%)$ & $22(19.1 \%)$ & $7(29.2 \%)$ & $12(12.5 \%)$ & $3(6.5 \%)$ & 0.04 & 0.06 & $\begin{array}{l}2.85 \\
(0.83-9.31)\end{array}$ \\
\hline
\end{tabular}

Values are presented as n (\%).

\#Data regarding comparisons between S. aureus, BHS, ANHS and Enterococci.

*Data regarding comparisons between BHS and ANHS.

Abbreviations: IE, infective endocarditis; BHS, beta-hemolytic streptococci; ANHS, alpha- or non-hemolytic streptococci; OR, odds ratio; CI,

confidence interval 